\title{
A Technology-assisted, High Intensive, Task-oriented Exercise Program to improve arm and hand functions in stroke patients: study protocol for the TecHITo feasibility and pilot trial.
}

\author{
Yih Wong MD, MSc, MAppSc ${ }^{1,2}$, Grethe Månum MD,PhD ${ }^{1,2}$, Birgitta Langhammer PT, PhD ${ }^{1,3}$ \\ 1 Research Department, Sunnaas Rehabilitation Hospital, Oslo, Norway \\ 2 Institute of Clinical Medicine, Faculty of Medicine, University of Oslo, Oslo, Norway \\ 3 Department of Physiotherapy, Faculty of Health Sciences, OsloMet - Oslo Metropolitan University, Oslo, Norway
}

\begin{abstract}
Background/purpose: Stroke patients with reduced upper limb functions often experience increased dependence in activities of daily living, restricted social participation, and low quality of life. In this context, an intervention, combining task-oriented exercises with a supportive device in the community rehabilitation unit and home environment, is hypothesized to increase perceived goal achievement, repetition, and intensity of exercises.
\end{abstract}

The primary objective is to evaluate the clinical usefulness of the program in terms of compliance and outcomes improvement.

The secondary objective is to compare the effectiveness of the program with or without the use of the device in a randomized controlled trial (RCT).

The project also combines technology and innovation by testing a biofeedback system that monitors compliances and encourages patients to practice with better quality of movements.

Methods/design: The study will first be carried out in a cohort of 30 stroke patients regardless of lesion location and temporal evolution. Subsequently, the pilot RCT will include 30 first-time stroke patients in which the experimental group will receive a supportive device for use during the exercises. The intervention will be performed at a rehabilitation unit 3 times/week for 3 weeks and at home 5 times/week for 9 weeks. Assessments will be performed at baseline, week 3 , and week 12 following intervention.
Primary outcome measure: Action Research Arm Test Secondary outcome measures: Fugl- Meyer Assessment, Motor Assessment Scale, Nine-peg-hole test, grip-strength, modified Ashworth Scale, EQ-5D instrument, and Borg scale.

Additionally, a qualitative evaluation will be performed.

Discussion: If the intervention proved effective, the proposed study will provide important information for clinicians and policy makers.

Trial registration: Clinicaltrials.gov, NCT03396939. Registered on 11 January 2018.

Keywords: Stroke, Rehabilitation, Task-oriented training, Orthosis, Motor recovery, Hand and arm function

\section{Inclusion criteria:}

- diagnosis of ischemic or hemorrhagic stroke;

- eligibility to cooperate in the study;

- age > 18 years;

- upper extremity hemiparesis with Motor Assessment Scale $\leq 3$ for arm and hand function.

\section{Exclusion criteria:}

- traumatic or nonvascular brain injury;

- subarachnoid or primary intraventricular hemorrhage;

- orthopedic-related/malfunctions in the arm and hand not related to stroke;

- severe co-morbidity;

- cognitive disorders with a Montreal Cognitive Assessment $(\mathrm{MoCa}) \leq 20)$. 